\title{
Atendimentos de emergência por violência em serviços públicos de Teresina, PI
}

\author{
Emergency assistance to violence in public services of Teresina, PI, Brasil
}

Atendimientos de emergencia por violencia en servicios publicos de Teresina, PI, Brasil

\author{
Márcio Dênis Medeiros Mascarenhas', Ana Amélia Galas Pedrosa" \\ 'Universidade Federal do Piauí. Teresina,PI \\ "Fundação Municipal de Saúde. Teresina, PI
}

Submissão: 19/03/2008

Aprovação: 3 1/07/2008

\section{RESUMO}

Este artigo objetivou descrever as características dos atendimentos de emergência por agressões e identificar fatores associados à sua ocorrência. A população de estudo incluiu vítimas de violência atendidas em dez serviços públicos de emergência de Teresina. Do total de atendimentos $(n=343)$, predominaram pacientes do sexo masculino $(76 \%)$, jovens $(40,9 \%)$, pardos $(63,2 \%)$ e com baixo nível de escolaridade $(42,4 \%)$. Os homens apresentaram maior chance de ter ingerido bebida alcoólica, sofrer agressão física por arma de fogo e objeto contundente em via pública. As mulheres tiveram maior chance de sofrer agressão psicológica e negligência no domicílio por familiares. O estudo apresenta o perfil da violência em Teresina e direciona o planejamento de políticas públicas de prevenção da violência e cultura de paz.

Descritores: Violência; Causas Externas; Vigilância; Epidemiologia.

\section{ABSTRACT}

This article aimed to describe the features of emergency assistance to aggressions and identify factors associated to their occurrence. The study population was victims of violence assisted in ten public emergency services in Teresina, PI, Brazil. Out of the 343 cases, there was a majority of male patients (76\%), young (40.9\%), pardos (63.2\%) and with low level of education (42.4\%). The men were more likely to have drunk alcohol, suffered physical aggression by firearm and blunt object on the streets. The women were more likely to have suffered psychological aggression and negligence by relatives at home. The study shows the violence profile in Teresina and gives directions to the management of public policies of violence prevention and culture of peace.

Descriptors: Violence; External Causes; Surveillance; Epidemiology.

\section{RESUMEN}

Este artículo buscó describir las características de los atendimientos de emergencia por agresiones y identificar factores asociados a su ocurrencia. La población de estudio incluyó victimas de violencia atendidas en diez servicios públicos de emergencia en Teresina, PI, Brasil. De un total de 343 atendimientos, predominaron pacientes del sexo masculino (76\%), jóvenes (40,9\%), pardos (63,2\%) y con bajo nivel de escolaridad (42,4\%). Los hombres presentaron mayor probabilidad de haber bebido alcohol, sufrido agresión física por arma de fuego y objeto contundente en vía pública. Las mujeres tuvieron mayor probabilidad de haber sufrido agresión psicológica y negligencia por parientes en casa. El estudio muestra el perfil de violencia en Teresina y da direcciones al planeamiento de políticas públicas de prevención de la violencia y cultura de paz.

Descriptores: Violencia; Causas Externas; Vigilancia; Epidemiología. 


\section{INTRODUÇÃO}

Embora apresente concepção complexa, a violência pode ser definida, segundo a Organização Mundial da Saúde (OMS), como o uso de força física ou poder, em ameaça ou na prática, contra si próprio, outra pessoa, um grupo ou comunidade, Que resulte ou possa resultar em lesão, morte, dano psicológico, desenvolvimento prejudicado ou privação ${ }^{(1)}$. Estima-se Que, a cada ano, os eventos violentos sejam responsáveis por mais de um milhão de mortes, a maior parte deles ocorridos entre pessoas em idade produtiva, sendo os homens as vítimas mais freqüentes ${ }^{(2-4)}$.

Considerando a abordagem da violência no campo da saúde pública, torna-se fundamental obter o maior número de conhecimentos possível sobre seus diferentes aspectos, a fim de reunir, sistematicamente, dados sobre a extensão, características e conseqüências desse problema de saúde pública em níveis local, nacional e internacional, dimensionando seu impacto na vida das pessoas.

Levantamento realizado por Schraiber et al ${ }^{(5)}$ demonstra Que a produção brasileira sobre violência e saúde no período de 1980 a 2005 segue a tendência internacional, na Qual os primeiros estudos tratam de dados de mortalidade por causas externas (acidentes e violência), caracterização da magnitude e importância dessas causas de morte em relação às demais, evoluindo, então, para a sistematização do entendimento da violência enQuanto processo de causas múltiplas Que se diferenciam e se articulam.

De acordo com os últimos dados disponíveis, as causas externas ocuparam a terceira posição entre as causas de morte no Brasil em 2005, totalizando 127.633 óbitos no país e 512 mortes em Teresina, capital do Piaú. Para fins de vigilância epidemiológica, o monitoramento das causas externas é realizado por meio da análise dos dados da declaração de óbito e da autorização de internação hospitalar na rede pública e em instituições conveniadas ao SUS, constituindo importante ferramenta para o conhecimento da carga da violência no Brasil $^{(6,7)}$. No entanto, esses dados limitam-se a caracterizar apenas os casos mais graves, cujo desfecho tenha sido o óbito ou a internação, impossibilitando o conhecimento das lesões menos graves, mas que são responsáveis por grande demanda de atendimentos nos serviços públicos de emergência.

Para superar essa falta de informações e em consonância com a Política Nacional de Redução da Morbimortalidade por Acidentes e Violências, o Ministério da Saúde implantou, em 2006, a Rede de Serviços Sentinelas de Vigilância de Violências e Acidentes (Rede VIVA), estruturada em dois componentes: 1) vigilância de violências e acidentes em emergências hospitalares e 2) vigilância das violências sexual, doméstica e/ou outras violências interpessoais ${ }^{(8,9)}$. Em Teresina, a vigilância em emergências hospitalares foi implantada, inicialmente, em um único serviço, sendo expandida, em 2007, para todas as unidades de urgência e emergência dos sistemas públicos municipal e estadual de saúde.

Reconhecendo a violência como importante problema de saúde pública e a necessidade de se obterem dados sobre os atendimentos de emergência nos serviços públicos de Teresina, desenvolveu-se este trabalho, cujos objetivos foram descrever as características dos atendimentos de emergência por violência e identificar fatores associados à sua ocorrência nos serviços Que integraram a Rede VIVA em Teresina, no ano 2007.

\section{METODOLOGIA}

Estudo inter-relacional de corte transversal, realizado em dez serviços públicos de emergência (dois estaduais e oito municipais) da Rede VIVA em Teresina. Adotou-se o procedimento de amostragem por conveniência, tendo sido incluídos na população de estudo todos os pacientes vítimas de violência atendidos no período de 1 a 30 de setembro de 2007.

A coleta de dados ocorreu em plantões de 12 horas e em turnos alternados, facilitando o trabalho de campo e permitindo estabelecer a representatividade dos atendimentos de cada serviço. Foram seguidas as recomendações do Ministério da Saúde Quanto à identificação dos serviços sentinelas, metodologia de coleta, aplicação dos Questionários, treinamento dos entrevistadores (acadêmicos de enfermagem/medicina) e supervisores da pesøuisa.

O instrumento de coleta de dados incluiu variáveis referentes à pessoa atendida (escolaridade, idade, meio de locomoção utilizado para chegar ao hospital, raça/cor da pele, sexo), ocorrência (hora e local da ocorrência, suspeita de ingestão de bebida alcoólica), tipo e meio de violência (agressões/maus tratos, tentativa de suicídio, intervenção legal) e dados do agressor (relação com a vítima).

A análise dos dados foi realizada por meio da distribuição de freeüências segundo as características dos casos de violência. As diferenças entre as categorias analisadas foram mensuradas pelos testes do Qui-Quadrado e exato de Fisher. Identificaram-se os potenciais fatores de risco associados à ocorrência de violência por meio da estimativa da odds ratio (OR) e seus respectivos intervalos de confiança de 95\% (IC95\%). Adotou-se o nível de significância estatística de $5 \%(p<0,05)$. No uso da variável raça/cor da pele, as categorias amarela e indígena foram excluídas, enQuanto pretos e pardos passaram a constituir a categoria negra. As demais variáveis foram convertidas em variáveis dicotômicas. Os dados foram tabulados e processados no programa Epi Info, versão 3.4. I. Neste trabalho, incluíram-se apenas os registros de atendimentos de emergência por causas violentas do tipo agressão/maus tratos.

Por se tratar de ação de vigilância epidemiológica de âmbito nacional, o projeto de peseuisa foi aprovado pela Comissão Nacional de Ética em PesQuisa (CONEP), Que recomendou a substituição do termo de consentimento livre e esclarecido pelo consentimento verbal, obtido do paciente ou seu responsável, garantindo-lhes total anonimato, privacidade e garantia de atendimento (Parecer $n^{\circ} 286$ / 2007).

\section{RESULTADOS}

Foram registrados 388 atendimentos de emergência por violência, dentre os Quais predominaram as agressões/maus tratos $(n=343$; $88,4 \%)$, seguidos das tentativas de suicídio $(n=39 ; 10,1 \%)$ e intervenções legais $(n=6 ; 1,5 \%)$.

A Tabela I apresenta as principais características dos atendimentos de emergência por agressões/maus tratos segundo o sexo da vítima. Do total de vítimas de agressão, 261 (76, 1\%) eram homens, sendo Que a faixa etária mais afetada incluiu pessoas com idade de 20 a 29 anos (40,9\%), seguida pelas faixas de 30 a 39 anos (17,5\%) e de 10 a 19 anos $(15,1 \%)$. Predominaram os atendimentos entre vítimas de cor parda $(63,2 \%)$ e aqueles que informaram terem concluído entre cinco e oito anos de estudo $(42,4 \%)$. 
Tabela 1. Características dos atendimentos de emergência por agressões/maus tratos segundo sexo da vítima. Teresina, 2007.

\begin{tabular}{|c|c|c|c|c|c|c|}
\hline \multirow{2}{*}{ Características } & \multicolumn{2}{|c|}{ Masculino } & \multicolumn{2}{|c|}{ Feminino } & \multicolumn{2}{|c|}{ Total } \\
\hline & $\mathrm{n}$ & $\%$ & $\mathrm{n}$ & $\%$ & $\mathrm{n}$ & $\%$ \\
\hline \multicolumn{7}{|l|}{ Faixa etária (anos) } \\
\hline $0-9$ & 21 & 8,2 & 13 & 16,0 & 34 & 10,1 \\
\hline $10-19$ & 42 & 16,4 & 9 & 11,1 & 51 & 15,1 \\
\hline $20-29$ & 106 & 41,4 & 32 & 39,5 & 138 & 40,9 \\
\hline $30-39$ & 48 & 18,8 & 11 & 13,6 & 59 & 17,5 \\
\hline $40-49$ & 21 & 8,2 & 10 & 12,3 & 31 & 9,2 \\
\hline $50-59$ & 8 & 3,1 & 3 & 3,7 & 11 & 3,3 \\
\hline$>60$ & 10 & 3,9 & 3 & 3,7 & 13 & 3,9 \\
\hline \multicolumn{7}{|l|}{ Raça/cor da pele } \\
\hline Parda & 174 & 66,9 & 47 & 57,3 & 216 & 63,2 \\
\hline Preta & 54 & 20,8 & 13 & 15,9 & 67 & 19,6 \\
\hline Branca & 32 & 12,3 & 22 & 26,8 & 54 & 15,8 \\
\hline \multicolumn{7}{|l|}{ Escolaridade (anos de estudo) } \\
\hline Nenhuma & 24 & 10,0 & 7 & 9,2 & 31 & 9,8 \\
\hline $1-4$ & 62 & 25,8 & 10 & 13,2 & 72 & 22,8 \\
\hline $5-8$ & 106 & 44,2 & 28 & 36,8 & 134 & 42,4 \\
\hline $9-11$ & 30 & 12,5 & 21 & 27,6 & 51 & 16,1 \\
\hline 12 e mais & 2 & 0,8 & 0 & 0,0 & 2 & 0,6 \\
\hline Não se aplica & 16 & 6,7 & 10 & 13,2 & 26 & 8,2 \\
\hline \multicolumn{7}{|c|}{ Meio de locomoção para chegar ao hospital } \\
\hline Ambulância ${ }^{\mathrm{a}}$ & 118 & 45,4 & 17 & 21,3 & 135 & 39,7 \\
\hline Veículo particular & 87 & 33,5 & 42 & 52,5 & 129 & 37,9 \\
\hline Viatura da polícia & 23 & 8,8 & 10 & 12,5 & 33 & 9,7 \\
\hline A pé & 20 & 7,7 & 7 & 8,8 & 27 & 7,9 \\
\hline Outro & 10 & 3,8 & 4 & 5,0 & 14 & 4,1 \\
\hline Coletivo & 2 & 0,8 & 0 & 0,0 & 2 & 0,6 \\
\hline \multicolumn{7}{|l|}{ Horário de ocorrência } \\
\hline $06: 00-17: 59$ & 88 & 33,9 & 34 & 42,5 & 122 & 35,9 \\
\hline $18: 00-05: 59$ & 171 & 66,1 & 46 & 57,5 & 217 & 64,1 \\
\hline \multicolumn{7}{|l|}{ Local de ocorrência } \\
\hline Via pública & 109 & 42,1 & 23 & 28,8 & 132 & 38,9 \\
\hline Residência & 62 & 24,0 & 40 & 50,0 & 102 & 30,1 \\
\hline Bar ou similar & 63 & 24,3 & 11 & 13,8 & 74 & 21,8 \\
\hline Trabalho & 7 & 2,7 & 5 & 6,3 & 12 & 3,6 \\
\hline Escola & 6 & 2,3 & 1 & 1,3 & 7 & 2,1 \\
\hline Local de lazer/esporte & 3 & 1,2 & 0 & 0,0 & 3 & 0,9 \\
\hline Outro & 9 & 3,5 & 0 & 0,0 & 9 & 2,7 \\
\hline \multicolumn{7}{|c|}{ Suspeita de ingestão de bebida alcoólica } \\
\hline Sim & 153 & 58,6 & 17 & 20,7 & 170 & 49,6 \\
\hline Não & 108 & 41,4 & 65 & 79,3 & 173 & 50,4 \\
\hline \multicolumn{7}{|l|}{ Tipo de agressão } \\
\hline Física & 246 & 94,3 & 68 & 82,9 & 314 & 91,5 \\
\hline Psicológica & 69 & 26,4 & 40 & 48,8 & 109 & 31,8 \\
\hline Negligência & 13 & 5,0 & 12 & 14,6 & 25 & 7,3 \\
\hline Sexual & 0 & 0 & 2 & 2,4 & 2 & 0,6 \\
\hline \multicolumn{7}{|l|}{ Meio de agressão } \\
\hline Força corporal & 132 & 50,6 & 45 & 54,9 & 177 & 51,6 \\
\hline Objeto perfurocortante & 102 & 39,1 & 27 & 32,9 & 129 & 37,6 \\
\hline Ameaça & 84 & 32,2 & 36 & 43,9 & 120 & 35,0 \\
\hline Objeto contundente & 57 & 21,8 & 9 & 11,0 & 66 & 19,2 \\
\hline Arma de fogo & 30 & 11,5 & 2 & 2,4 & 32 & 9,3 \\
\hline Objeto/substância Quente & 3 & 1,1 & 1 & 1,2 & 4 & 1,2 \\
\hline Envenenamento & 2 & 0,8 & 0 & 0 & 2 & 0,6 \\
\hline \multicolumn{7}{|l|}{ Provável autor da agressão } \\
\hline Familiar & 37 & 14,2 & 33 & 40,2 & 70 & 20,4 \\
\hline Conhecido & 126 & 48,3 & 40 & 48,8 & 166 & 48,4 \\
\hline Desconhecido & 88 & 33,7 & 7 & 8,5 & 95 & 27,7 \\
\hline
\end{tabular}


Tabela 2. Análise das variáveis associadas à ocorrência de atendimentos por agressões/maus tratos segundo sexo da vítima. Teresina, 2007.

\begin{tabular}{|c|c|c|c|c|c|c|}
\hline \multirow{2}{*}{ Variáveis } & \multicolumn{2}{|c|}{ Masculino } & \multicolumn{2}{|c|}{ Feminino } & \multirow{2}{*}{ OR (IC95\%) } & \multirow[b]{2}{*}{$\mathrm{p}$} \\
\hline & $\mathrm{n}$ & $\%$ & $\mathrm{n}$ & $\%$ & & \\
\hline \multicolumn{7}{|l|}{ Raça/cor da pele } \\
\hline Negra (parda+preta) & 228 & 87,7 & 60 & 73,2 & 2,61 & $<0,0$ I \\
\hline Branca & 32 & 12,3 & 22 & 26,8 & $(1,35-5,03)$ & \\
\hline \multicolumn{7}{|c|}{ Escolaridade (anos de estudo) } \\
\hline$<8$ & 192 & 86,2 & 45 & 68,2 & 2,8 & $<0,01$ \\
\hline$>8$ & 32 & 13,8 & 21 & 31,8 & $(1,41-5,56)$ & \\
\hline \multicolumn{7}{|c|}{ Agressão ocorreu no domicílio } \\
\hline Sim & 62 & 23,9 & 40 & 50,0 & 0,31 & $<0,001$ \\
\hline Não & 197 & 76,1 & 40 & 50,0 & $(0,18-0,55)$ & \\
\hline \multicolumn{7}{|c|}{ Suspeita de ingestão de bebida alcoólica } \\
\hline $\operatorname{Sim}$ & 153 & 58,6 & 17 & 20,7 & 5,42 & $<0,00$ I \\
\hline Não & 108 & 41,4 & 65 & 79,3 & $(2,91-10,20)$ & \\
\hline \multicolumn{7}{|l|}{ Agressão física } \\
\hline Sim & 246 & 94,2 & 68 & 82,9 & 3,38 & $<0,0$ I \\
\hline Não & 15 & 5,8 & 14 & 17,1 & $(1,45-7,83)$ & \\
\hline \multicolumn{7}{|l|}{ Agressão psicológica } \\
\hline Sim & 69 & 26,4 & 40 & 48,8 & 0,38 & $<0,001$ \\
\hline Não & 192 & 73,6 & 42 & 51,2 & $(0,22-0,65)$ & \\
\hline \multicolumn{7}{|l|}{ Negligência } \\
\hline Sim & 13 & 5,0 & 12 & 14,6 & 0,31 & $<0,01$ \\
\hline Não & 248 & 95,0 & 70 & 85,4 & $(0,12-0,75)$ & \\
\hline \multicolumn{7}{|l|}{ Objeto contundente } \\
\hline Sim & 57 & 21,8 & 9 & 11,0 & 2,27 & 0,04 \\
\hline Não & 204 & 78,2 & 73 & 89,0 & $(1,02-5,18)$ & \\
\hline \multicolumn{7}{|l|}{ Arma de fogo } \\
\hline Sim & 30 & 11,5 & 2 & 2,4 & 5,19 & 0,02 \\
\hline Não & 231 & 88,5 & 80 & 97,6 & $(1,17-32,18)$ & \\
\hline \multicolumn{7}{|c|}{ Provável autor da agressão era um familiar } \\
\hline Sim & 37 & 14,2 & 33 & 40,2 & 0,25 & $<0,001$ \\
\hline Não & 224 & 85,8 & 49 & 59,8 & $(0,13-0,45)$ & \\
\hline
\end{tabular}

Em busca de atendimento nos serviços de emergência, os meios mais freeüentemente utilizados para o transporte das vítimas foram ambulâncias $(39,7 \%)$ e veículos particulares (37,9\%). Quanto ao horário de ocorrência do evento violento, observou-se maior freqüência entre o início da noite e final da madrugada, Quando ocorreram $64,1 \%$ das agressões registradas. No Que diz respeito ao local de ocorrência, destacaram-se os seguintes cenários: via pública (ruas, rodovias, praças), com 38,9\%; ambiente domiciliar (residência da vítima ou de terceiros), com $30,1 \%$, e bares ou similares, com 21,8\%.

Em Quase metade dos atendimentos (49,6\%), suspeitou-se de ingestão de bebida alcoólica pela vítima. Quanto ao tipo de agressão, predominaram agressão física $(91,5 \%)$ e agressão psicológica $(31,8 \%)$. Foram registrados apenas dois casos de violência sexual entre as mulheres. Dentre os meios utilizados para efetivar o ato agressivo, destacaram-se o uso de força corporal/espancamento $(51,6 \%)$ e objetos perfurocortantes (37,6\%), seguidos de agressão verbal/ ameaça $(35,0 \%)$ e objetos contundentes $(19,2 \%)$. Agressões por arma de fogo foram mais freeüentes entre os homens (1 1,5\%).

Quanto ao tipo de relação entre o provável autor da agressão e a pessoa atendida, os conhecidos (amigos, namorados, colegas de escola/trabalho) predominaram em ambos os sexos $(48,4 \%)$. Pessoas totalmente desconhecidas da vítima foram mais freqüentes entre os homens, enQuanto as mulheres foram agredidas em maior proporção por pessoas com as Quais tinham relações familiares, como cônjuge, mãe, pai, padrasto, madrasta, filhos, irmãos, netos, sobrinhos, primos, dentre outros (Tabela 1).

A Tabela 2 apresenta a estimativa de chance de ocorrência de agressões entre homens e mulheres conforme variáveis demográficas e referentes ao tipo de agressão. Pode-se observar Que houve diferença estatisticamente significativa na ocorrência de agressões entre homens de pele negra $(\mathrm{OR}=2,61$; IC $95 \%=1,35-5,03$; $\mathrm{p}<0,0 \mathrm{I})$ e com menor escolaridade $(\mathrm{OR}=2,8$; IC95\% $=1,4 \mathrm{I}-5,56$; $\mathrm{p}<0,0 \mathrm{l})$ Quando comparados às mulheres. A chance de os homens se encontrarem sob suspeita de uso de bebida alcoólica foi 5,42 vezes maior em relação às mulheres (IC95\%=2,91-10,20; $\mathrm{p}<0,001$ ). Os homens também apresentaram maior chance de sofrerem agressão física $(O R=3,38 ; \mathrm{IC} 95 \%=1,45-7,83 ; \mathrm{p}<0,01)$ envolvendo uso de arma de fogo $(\mathrm{OR}=5,19 ; \mathrm{IC} 95 \%=1,17-32,18$; $p=0,02)$ e objeto contundente $(O R=2,27$; IC95\%=1,02-5, 18 ; $\mathrm{p}=0,04)$. Por outro lado, a proporção de vítimas de agressão psicológica $(48,8 \%)$ e negligência (14,6\%) foi maior entre as mulheres, apresentando diferença estatisticamente significativa em comparação ao Que se observou entre os homens.

Quanto ao local de ocorrência, o domicílio foi identificado como ambiente desfavorável para as mulheres, pois nesse cenário elas 
foram mais freQüentemente agredidas em relação aos homens, para os Quais o espaço domiciliar configurou-se como fator de proteção $(O R=0,31 ; I C 95 \%=0,18-0,55 ; p<0,001)$. Além disso, as mulheres encontraram-se sob maior risco de serem agredidas por seus próprios familiares, na proporção de $40,2 \%$, contra $14,2 \%$ entre os homens, sendo essa diferença estatisticamente significativa $(O R=0,25 ; \mathrm{IC} 95 \%=0,13-0,45 ; \mathrm{p}<0,00 \mathrm{I})$ (Tabela 2).

\section{DISCUSSÃO}

A abordagem da saúde pública direcionada ao problema da violência está baseada em Quatro etapas: i) conhecimentos básicos sobre a violência (extensão, características, conseqüências); ii) causas e fatores Que aumentam ou diminuem o risco de violência; iii) proposição de formas de prevenção da violência (planejamento, monitoramento, avaliação) e iv) disseminação de intervenções promissoras e informações sobre custo e eficácia dos programas implantados ${ }^{(1)}$. Dessa forma, a vigilância epidemiológica de violências constitui atividade relevante para a sociedade, pois, além de permitir o monitoramento e a análise de possíveis mudanças no perfil desses agravos, contribui para a educação da população e o planejamento de ações intersetoriais de prevenção ${ }^{(2)}$.

No Brasil, a abordagem da violência no âmbito da saúde pública encontra-se nas etapas iniciais, buscando aproximar-se mais do conhecimento das características e causas da violência, bem como da identificação de seus fatores de risco. Essas informações, primordiais para o desenvolvimento de ações locais, no entanto, continuam inacessíveis para tomadores de decisão, Quer seja por limitações no atual modelo de vigilância, Quer seja pela intrincada rede de fatores Que favorecem o silêncio acerca da temática violência.

Neste estudo, os homens representaram a maior proporção de vítimas de agressão entre os atendimentos de emergência. Em 2005 , foram registrados 47.578 óbitos por agressão no Brasil, dos Quais 92\% foram de homens, resultando em taxa de mortalidade de 48,2/ 100.000 homens, valor 12 vezes maior do Que a taxa observada entre as mulheres ${ }^{(7)}$. $\mathrm{O}$ fato de os homens consituírem a maior parcela de vítimas de agressão não pode ser justificado apenas por um fator biológico específico. Para Souza ${ }^{(10)}$, a maior ocorrência de agressões entre os homens pode estar relacionada com um dos grandes símbolos de masculinidade no mundo atual - as armas, Que materializam o poder de submeter o outro a seus desejos e interesses (poder de vida ou morte), por meio de objetos Que são introduzidos desde cedo na vida do menino, na forma de brinquedos, e passam a fazer parte do universo masculino. Todavia, os resultados deste estudo também apontam para o impacto da violência no sexo feminino, sendo Que a violência torna a mulher mais vulnerável ao desenvolvimento de problemas físicos (principalmente Quando se trata de violência física ou sexual), interpessoais, familiares e sociais, como resultado da permanente situação de estresse e da falta de esperança em mudar sua condição de vítima ${ }^{(1)}$.

Quanto à faixa etária, adolescentes e adultos jovens têm sido freqüentemente identificados como as principais vítimas de violência $^{(7,12)}$, o Que pode ser explicado por fatores como inexperiência, busca de emoções, prazer em experimentar situações de risco, impulsividade e abuso de substâncias psicoativas ${ }^{(13,14)}$. Crianças e adolescentes são os dois grupos mais vulneráveis a sofrerem violação de seus direitos, afetando direta e indiretamente sua saúde física, mental e emocional, o Que reQuer prestação do atendimento adeQuado, alívio do sofrimento e modos de prevenção sob um modelo ampliado de assistência à saúde ${ }^{(15,16)}$.

De acordo com dados divulgados pelo Ministério da Saúde ${ }^{(17)}$, a população negra apresenta os maiores coeficientes de mortalidade por causas externas. No período de 2000 a 2003, esses coeficientes mantiveram-se estáveis entre os brancos, enQuanto, entre a população parda e preta, observou-se aumento no risco de morte por causas violentas. Embora um grupo social não se defina por relações de raça ou cor, diferenças étnicas associam-se a desigualdades sociais e condicionam a forma de viver e de morrer de grupos de pessoas. A etnia em si não é um fator de risco, mas a inserção social adversa de um grupo racial/étnico se constitui em característica de vulnerabilidade $^{(18)}$. Outro aspecto importante, Que sozinho não explica a ocorrência da violência, foi a considerável proporção de atendimentos de pessoas com baixo nível de escolaridade, resultado semelhante ao apresentado em outros estudos nacionais ${ }^{(4,18,19)}$, nos Quais também se verificou relação inversa entre a ocorrência de violências e o número de anos de estudo da vítima.

O meio de locomoção mais utilizado para chegar aos serviços de emergência foi a ambulância, destacando-se as unidades de resgate do SAMU, principal componente da Política Nacional de Atenção às Urgências (PNAU) do Ministério da Saúde. As principais ações dessa política incluem: a) organização do atendimento de urgência em unidades de pronto-atendimento; b) estruturação do atendimento pré-hospitalar móvel (SAMU/192); c) reorganização das grandes urgências e prontos-socorros em hospitais; d) criação de retaguarda hospitalar para os atendimentos de urgência e e) estruturação do atendimento pós-hospitalar ${ }^{(8)}$.

A literatura assinala os espaços públicos (ruas, bares e outros locais públicos) como os principais cenários onde se registra a ocorrência de eventos violentos, especialmente Quando envolvem agressores e vítimas do sexo masculino, eneuanto a maioria das ações violentas contra a mulher ${ }^{(10)}$ ocorre no espaço privado do lar. Esse ambiente foi o segundo local em Que grande parte dos eventos violentos tomou lugar, revelando-se o mais favorável à ocorrência de agressões e abusos contra crianças e mulheres. Pode-se aventar Que, por permanecerem mais tempo em seus lares, crianças, adolescentes e mulheres acabam sendo violentadas mais freeüentemente nos domicílios $^{(15)}$.

É comprovada a associação entre o consumo de bebidas alcoólicas e a ocorrência de eventos violentos, uma vez Que o álcool atua, freeüentemente, como um desinibidor, facilitando a violência ${ }^{(20,21)}$. Em Cali, na Colômbia, Quase metade das vítimas atendidas em unidades de pronto-atendimento encontrava-se sob suspeita ou referiu estar sob influência do álcool ${ }^{(22)}$. Esses achados assemelham-se aos resultados obtidos em Teresina, principalmente entre as vítimas do sexo masculino, corroborando, assim, a influência do álcool na ocorrência de eventos violentos.

Evidencia-se a maior participação de mulheres como vítimas da violência Quando se trata de agressão psicológica, sexual ou por negligência, a despeito do pequeno número de atendimentos por esse tipo de violência. Tais achados são semelhantes aos resultados obtidos em Guarulhos, São Paulo ${ }^{(23)}$, e Feira de Santana, Bahia ${ }^{(15)}$. A negligência é um importante problema social, caracterizado pela omissão em prover as necessidades e cuidados básicos para o desenvolvimento físico, emocional e social da pessoa, merecendo destaque a proporção de 
atendimentos com relatos de violência psicológica ou sexual, cuja ocorrência foi predominante entre as mulheres.

A violência praticada contra o sexo feminino é conhecida como violência de gênero, porQue se relaciona à condição de subordinação da mulher na sociedade, incluindo-se nessa categoria a agressão física, a sexual, a psicológica e a econômica. Estima-se que de 10\% a $50 \%$ das mulheres em todo o mundo já sofreram alguma forma de violência perpetrada por seus parceiros íntimos, um fenômeno Que vem sendo encarado como importante problema de saúde pública. Em estudo realizado entre usuárias de serviços públicos de saúde, $45 \%$ das mulheres referiram ocorrência de Qualquer tipo de violência pelo menos uma vez em suas vidas ${ }^{(24)}$.

Com relação ao autor do evento violento, destaca-se a maior participação de agressores totalmente desconhecidos Quando a vítima era do sexo masculino. Quanto às vítimas do sexo feminino, o agressor era, predominantemente, um membro da família. Esses resultados podem ser explicados pela maior ocorrência de violência contra homens nos espaços públicos, onde estão os agressores com relações extrafamiliares com a vítima, en@uanto o espaço privado do domicílio é mais propício para a ocorrência de abuso sexual, negligência e violência psicológica, perpretados por pais, cônjuges e/ou cuidadores com relações familiares com a vítima, a Qual, na maior parte das vezes, é do sexo feminino ${ }^{(10,15)}$.

\section{CONSIDERAÇÕES FINAIS}

O estudo apresenta o perfil dos atendimentos de emergência por violência, identificando os fatores de risco associados à sua ocorrência. Os aspectos apresentados servem para se direcionar um olhar cuidadoso aos diversos sinais e sintomas Que somatizam situações de sofrimento. Trata-se de uma modalidade de vigilância de violências em serviços sentinelas Que disponibiliza dados em tempo hábil, de forma Que sua análise e interpretação proporcionem as bases para a tomada de decisões.

Nesse sentido, reconhecer fatores de riscos para construir mecanismos de prevenção à violência e promoção da saúde é o desafio Que se coloca para a prática interdisciplinar e multiprofissional em saúde, reduzindo a invisibilidade da violência sofrida e Que chega aos serviços de emergência, muitas vezes, camuflada. Ao percebê-la, o profissional de saúde pode exercer o papel de facilitador da constituiçao de uma rede de proteção às vitimas de violência.

Agradecemos o apoio dos dirigentes da Fundação Municipal de Saúde de Teresina, diretores e funcionários dos hospitais onde ocorreu a pesquisa, equipe de execução (supervisores, coletadores, digitadores) e o fundamental suporte técnico-financeiro da Coordenaçao Geral de Vigilância de Doenças e Agravos Não Transmissíveis do Ministério da Saúde (CGDANT/MS).

\section{REFERÊNCIAS}

I. World Health Organization. World report on violence and health. Geneva (SWT): WHO; 2002.

2. Dahlberg LL, Krug EG. Violence a global public health problem. Ciênc Saúde Coletiva 2006; 1 1:277-92.

3. Minayo MCS. Violência: um problema para a saúde dos brasileiros. In: Ministério da Saúde. Impacto da violência na saúde dos brasileiros. Brasília (DF): Ministério da Saúde; 2005. p. 9-42.

4. Soares Filho AM, Souza MFM, Gazal-Carvalho C, Malta DC, Alencar AP, Silva MMA, et al. Análise da mortalidade por homicídios no Brasil. Epidemiol Serv Saúde 2007; 16: 7- 18.

5. Schraiber LB, D’Oliveira AFPL, Couto MT. Violência e saúde: estudos científicos recentes. Rev Saúde Pública 2006;40(esp): 112-20.

6. Malta DC, Cezário AC, Moura L, Morais Neto OL, Silva Jr JB. A construção da vigilância e prevenção das doenças crônicas não transmissíveis no contexto do Sistema Único de Saúde. Epidemiol Serv Saúde 2006; 1 5: 47-65.

7. Ministério da Saúde. Departamento de Informática do SUS. Informações de saúde. Brasília (DF); Ministério da Saúde; 2008 [citado em 10 abr 2008]. Disponível em: URL: http:// www.datasus.gov.br

8. Malta DC, Lemos MSA, Silva MMA, Rodrigues EMS, GazalCarvalho C, Morais Neto OL. Iniciativas de vigilância e prevenção de acidentes e violências no contexto do Sistema Único de Saúde (SUS). Epidemiol Serv Saúde 2007; 16: 45-55.

9. Gawryszewski VP, Silva MMA, Malta DC, Mascarenhas MDM, Costa VC, Matos SG, et al. A proposta da rede de serviços sentinela como estratégia de vigilância de violências e acidentes. Ciênc Saúde Coletiva 2006: I I (sup): 1269-78.

10. Souza ER. Masculinidade e violência no Brasil: contribuições para a reflexão no campo da saúde. Ciênc Saúde Coletiva 2005; 10: 59-70.

11. Reichenheim ME, Dias AS, Moraes CL. Co-ocorrência de violência física conjugal e contra filhos em serviços de saúde. Rev Saúde Pública 2006; 40: 595-603.

12. Souza ER, Minayo MCS, Cavalcante FG. The impact of suicide on morbidity and mortality in the population of Itabira. Ciênc Saúde Coletiva 2006; 11: 409-18.

13. Deslandes SF, Assis SG, Santos NC. Violências envolvendo crianças no Brasil: um plural estruturado e estruturante. In: Ministério da Saúde. Impacto da violência na saúde dos brasileiros. Brasília (DF): Ministério da Saúde; 2005. p. 43-78.

14. Ministério da Saúde. Saúde Brasil 2006: uma análise da desigualdade em saúde. Brasília (DF): Ministério da Saúde; 2006.

15. Costa COM, Carvalho RC, Bárbara JFRS, Santos CAST, Gomes WA, Sousa HL. O perfil da violência contra crianças e adolescentes, segundo registros de Conselhos Tutelares: vítimas, agressores e manifestações de violência. Ciênc Saúde Coletiva 2007; 12: II29-41.

16. Souza ER, Mello Jorge MHP. Impacto da violência na infância e adolescência brasileiras: magnitude da morbimortalidade. In: Ministério da Saúde. Violência faz mal à saúde. Brasília (DF): Ministério da Saúde; 2006. p. 23-8.

17. Ministério da Saúde. Saúde Brasil: uma análise da situação de saúde. Brasília (DF): Ministério da Saúde; 2005.

18. Batista LE, Escuder MML, Pereira JCR. A cor da morte: causas de óbito segundo características de raça no Estado de São Paulo, 1999 a 2001. Rev Saúde Pública 2004;38: 630-6.

19. Minayo MCS, Souza ER. Violência para todos. Cad Saúde Pública 1993;9:65-78.

20. Castillo MMA, Caufield C, Gómez Meza MV. Consumo de drogas 
y violência laboral em mujeres trabajadoras de Monterrey, N.

L., México. Rev Latino-Am Enfermagem 2005; 13 (esp): 11 64-8.

21. Zilberman ML, Blume SB. Violência doméstica, abuso de álcool e substâncias psicoativas. Rev Bras Psieuiatr 2005; 27(sup): S5I-S55.

22. Castro MB, Rendón LF, Rojas MC, Durán CA, Albornoz M. Caracterización de los pacientes con lesiones de causa externa mediante un sistema de vigilancia epidemiológica. Rev Colomb Cir 2006; $21: 180-9$.

23. Silva MAI, Ferriani MGC. Domestic violence: from the visible to the invisible. Rev Latino-Am Enfermagem 2007; 15: 275-81.

24. Marinheiro ALV, Vieira EM, Souza L. Prevalência da violência contra a mulher usuária de serviço de saúde. Rev Saúde Pública 2006; 40: 604-10. 